(XIV b) erkennt. Von besonderem Interesse sind jedoch die Reaktionen XIII $\mathrm{c}$ und XIV c, in denen sich das $\mathrm{O}^{-}$-Ion an die aromatische Verbindung anlagert unter gleichzeitiger Abspaltung eines Wasserstoffatoms. Den entstehenden negativen Ionen dürfte die besonders stabile Struktur von Phenolat-Anionen zuzuschreiben sein. Unter diesen Umständen wäre zu schließen, daß die Stärke der C-O-Bindung im Phenolat-Anion größer ist als die der $\mathrm{C}-\mathrm{H}$-Bindung im Benzol. Mit D $\left(\mathrm{C}_{6} \mathrm{H}_{5}-\mathrm{H}\right)=102 \mathrm{kcal} / \mathrm{mol}^{11}$ erhält man

$$
\mathrm{D}\left(\mathrm{C}_{6} \mathrm{H}_{5}-\mathrm{O}^{-}\right) \geqq 4,4 \mathrm{eV} .
$$

Der Deutschen Forschungsgemeinschaft danken wir für die Unterstützung dieser Untersuchungen.

\title{
Diffusion von Tritium in Quarz und Quarzglas
}

\author{
HJ. Matzke \\ Institut für Kern- und Radiochemie der Technischen Hochschule Braunschweig \\ (Z. Naturforschg. 22 a, 965-969 [1967] ; eingegangen am 3. März 1967)
}

\begin{abstract}
The release of tritium from single crystalline quartz and from fused silica has been measured following $40 \mathrm{keV}$ ion bombardment to a dose of $4 \times 10^{11}$ ions $/ \mathrm{cm}^{2}$. The release can be explained by normal volume diffusion with $D_{0}=10^{1 \pm 1} \mathrm{~cm}^{2} \mathrm{sec}^{-1}$ for both materials and activation enthalpies of $(42 \pm 5) \mathrm{kcal} / \mathrm{mole}$ for quartz and $(52 \pm 5) \mathrm{kcal} / \mathrm{mole}$ for fused silica. Release from quartz is faster than release from fused silica. This latter diffusion process, however, is essentially slower than diffusion of hydrogen during permeation. Possible reasons for this discrepancy are the existence of radiation damage due to the ion bombardment or the chemical state of hydrogen which cause a different elementary process for mass transport: Hydrogen diffuses in molecular form during permeation whereas probably tritium atoms or ions will diffuse following ion bombardment.
\end{abstract}

Die Diffusion von Wasserstoff und anderen leichten Gasen, bes. Helium, in Quarzglas wurde seit mehreren Jahrzehnten mittels der sog. Permeationsmethode untersucht. Man mißt dazu die Gleichgewichtsdurchlässigkeit von platten- oder zylinderförmigen Proben, die einen Gasvorrat unter einem veränderlichen Druck von Hochvakuum trennen. Das Gas wird an Hand des Druckanstiegs auf der Vakuumseite oder, besser, mittels Massenspektrometer nachgewiesen. Der Permeationsvorgang schließt somit verschiedene Schritte ein: Adsorption und evtl. Dissoziation des Gases auf der Druckseite, Lösung des Gases in der Probe, Diffusion im Konzentrationsgradienten durch die Probe, Übergang in eine Oberflächenschicht auf der Vakuumseite, evtl. Rekombination der Dissoziationsprodukte und endlich Desorption von der zweiten Oberfläche. Jeder einzelne dieser Prozesse muß also mindestens so schnell wie der Gesamtpermeationsvorgang verlaufen. Durch Verfolgen der Druckabhängigkeit der Permeationskonstanten $P$ kann gezeigt werden, daß Wasserstoff atomar durch Metalle, wie z.B. Eisen, diffundiert,

1 R. M. Barrer, Diffusion in and through Solids, Cambridge University Press 1941.

2 N. W. Taylor u. W. Rost, J. Chem. Phys. 6, 612 [1938].

3 G. A. Williams u. J. B. Ferguson, J. Am. Chem. Soc. 44, 2160 [1922]. während $\mathrm{H}_{2}$-Moleküle durch Quarzglas diffundieren.

Bei bekannter Löslichkeit $s$ läßt sich aus der Permeationskonstanten $P$ die Diffusionskonstante $D$ berechnen gemäß

$$
P=s D .
$$

Für Quarzglas wurde eine schnelle Diffusion von $\mathrm{H}_{2}$ und $\mathrm{He}$ festgestellt mit präexponentiellen Faktoren $D_{0}$ von etwa $10^{-4 \pm 1} \mathrm{~cm}^{2} \mathrm{sec}^{-1}$ und Aktivierungsenthalpien von $6-10 \mathrm{kcal} / \mathrm{Mol}$, wobei die Diffusionsrate von He bei mittleren Temperaturen $(600$ bis $700{ }^{\circ} \mathrm{C}$ ) etwa $30-50 \mathrm{mal}$ größer als die von Wasserstoff war ${ }^{1-5}$. Die Permeation durch Quarzkristalle verlief wesentlich langsamer.

In letzter Zeit wurde verschiedentlich die Technik des Ionenbeschusses zur Einführung von Gasen in Festkörper benutzt, um anschließend die Diffusion des Gases zu untersuchen ${ }^{6}$. In diesem Fall wird das Gas also gewaltsam in den Probekörper eingebracht und befindet sich in oder nahe einer strahlengeschädigten Zone. Somit kann die Beweglichkeit des Gases durch Existenz oder Ausheilen von Strahlenschäden

${ }^{4}$ F. J. Norton, J. Am. Ceram. Soc. 36, 90 [1953].

5 D. E. Swets, R. W. Lee u. R. C. Frank, J. Chem. Phys. 34, 17 [1961].

${ }^{6}$ Zum Beispiel R. Kelly u. HJ. Matzke, J. Nucl. Mater. 17, 179 [1966]. 
, 

Nıus-Gleichungen beschrieben werden, wobei $D_{0}$ für beide Materialien $10^{1 \pm 1} \mathrm{~cm}^{2} \mathrm{sec}^{-1}$ beträgt und $\Delta H$ $=(42 \pm 5) \mathrm{kcal} / \mathrm{Mol}$ ist für Quarzkristalle und $(52 \pm 5) \mathrm{kcal} / \mathrm{Mol}$ für Quarzglas. Die nach den beiden im vorstehenden Abschnitt angegebenen Methoden ermittelten $\Delta H$-Werte stimmen innerhalb der Fehlergrenzen von $\pm 5 \mathrm{kcal} / \mathrm{Mol}$ überein.

\section{Diskussion}

Die S-förmige Gestalt der beiden Abgabekurven und die ,idealen“ $D_{0}$-Werte deuten darauf hin, da $\beta$ in beiden Fällen ein Gitterdiffusionsprozeß die $\mathrm{Ab}$ gabe bestimmt. Ein Zusammenhang der Gasabgabe mit einer der Phasenumwandlungen im $\mathrm{Si}-\mathrm{O}$ System ist nicht wahrscheinlich, da Quarzglas erst ab etwa $1000{ }^{\circ} \mathrm{C}$ rekristallisiert und die einzige Phasenumwandlung von Quarzkristallen im Temperaturbereich der Gasabgabe $\left(300-700{ }^{\circ} \mathrm{C}\right)$, nämlich der Übergang von $\alpha$ - zu $\beta$-Quarz bei $573{ }^{\circ} \mathrm{C}$, ein sehr schneller, diffusionsloser Vorgang ist (z. B. $\left.{ }^{8}\right)$. Ebenso erscheint es unwahrscheinlich, daß eine beschußbedingte Phasenumwandlung oder ein die Proben als Ganzes beeinflussender Strahlenschaden oder dessen Ausheilung die Tritiumabgabe bewirkte. Die die Proben treffende Gesamtionendosis betrug etwa $7 \times 10^{13}\left(\mathrm{~T}_{2}+\mathrm{Li}\right)$-Ionen $/ \mathrm{cm}^{2}$. Von Ionenbeschußversuchen an Quarzglas und Quarzkristallen mit $40 \mathrm{keV}$. Xe-Ionen ${ }^{8}$ ist bekannt, da $\beta$ eine Dosis von $4 \times 10^{13}$ Xe-Ionen $/ \mathrm{cm}^{2}$ eine Phasenumwandlung der Quarzkristalle zu einer quasi-amorphen Phase einleitet, die bei einer Dosis von $2 \times 10^{16} \mathrm{Xe}$-Ionen $/ \mathrm{cm}^{2}$ vollendet ist. Gleichzeitig wird Quarzglas durch den Beschuß in einer ähnlichen Weise geschädigt, so daß beide Materialien in eine „bestrahlungs-induzierte quasiamorphe Phase" überführt werden, die in einem engen Temperaturintervall zwischen etwa 625 und $750{ }^{\circ} \mathrm{C}$ in die ursprüngliche Phase zurückverwandelt wird. Gleichzeitig wird das meiste Gas freigesetzt. Die Gasabgabekurven sind also sehr steil und praktisch identisch für Quarzkristalle und Quarzglas. Im Gegensatz dazu zeigt sich in Abb. 2 für die Abgabe von Tritium ein Unterschied zwischen Quarzkristallen und Quarzglas. Außerdem sind beide Abgabekurven nicht steil, sondern erstrecken sich über einen Temperaturbereich von etwa $300{ }^{\circ} \mathrm{C}$, die Abgabe verläuft bei wesentlich niedrigeren Temperaturen

16 W. Primak, J. Appl. Phys. 34, 3630 [1963].

17 C. J. Beevers u. R. S. Nelson, Phil. Mag. 8, 1189 [1963]. und ist bei $625{ }^{\circ} \mathrm{C}$, dem Einsatzpunkt der Umwandlung der strahleninduzierten Phase, fast beendet. Schließlich wurde verschiedentlich gezeigt ${ }^{16,17}$, daß leichte Teilchen wesentlich weniger Strahlenschädigung verursachen als schwere Teilchen. Folglich erscheint es wahrscheinlich, daß Tritium durch einen Diffusionsprozeß freigesetzt wird.

Allerdings verläuft die Abgabe von Tritium aus Quarzglas wesentlich langsamer als die aus Permeationsmessungen ermittelte Diffusion von Wasserstoff. Eine solche Diffusion mit $D_{0} \approx 10^{-4 \pm 1}$ $\mathrm{cm}^{2} \mathrm{sec}^{-1}$ und $\Delta H=6-10 \mathrm{kcal} / \mathrm{Mol}$ würde bei den hier vorliegenden geometrischen Verhältnissen schon zwischen Zimmertemperatur und etwa $250{ }^{\circ} \mathrm{C}$ zur Vollendung gehen, während in der vorliegenden Arbeit die Abgabe von Tritium erst oberhalb $250{ }^{\circ} \mathrm{C}$ beginnt. Das Verhältnis von Diffusionskoeffizienten, ermittelt aus Permeationsmessungen, zu Diffusionskoeffizienten, gemessen nach Ionenbeschuß, beträgt etwa $10^{5 \pm 2}$ bei $500{ }^{\circ} \mathrm{C}$ und $10^{2 \pm 1}$ bei $800{ }^{\circ} \mathrm{C}$. Diese Werte können nicht durch den Massenunterschied von Wasserstoff und Tritium erklärt werden, da dieser durch die Wurzel aus dem Verhältnis der Massen bestimmt ist und somit nur 1,7 beträgt.

Eine bessere Erklärungsmöglichkeit bietet die gewaltsame Einführung des Tritiums. Während in Permeationsmessungen Wasserstoff als Molekül, vermutlich durch die Kanäle der Gitterstruktur, wandert, tritt Tritium bei Ionenbeschuß wahrscheinlich ato$\operatorname{mar}^{12}$ in die Probe ein. Die verwendete Ionendosis ist so klein, daß eine Rekombination der Tritium. atome zu Molekülen unwahrscheinlich erscheint. Somit könnte für das (evtl. ionisierte) atomare Tritium ein anderer Diffusionsmechanismus vorliegen als für neutrale Wasserstoffmoleküle. Darauf deutet auch der Befund hin, daß die Abgabe aus Quarzglas, das die offenere Struktur besitzt, langsamer verläuft. Eine langsamere Abgabe aus Quarzglas wurde ebenfalls für die Gitterdiffusion von Xenon ${ }^{8}$ nach Ionenbeschuß gefunden, während die Sauerstoffselbstdiffusion ${ }^{18,19}$ in Quarzglas schneller als in Quarzkristallen verläuft. Alle diese Diffusionsvorgänge verlaufen jedoch wesentlich langsamer als die hier beobachtete Abgabe von Tritium. Die Selbstdiffusion von Si wurde bisher noch nicht gemessen, sollte aber ebenfalls langsamer verlaufen. Somit scheint ein eigener Diffusionsvorgang für Tritium zu existieren.

18 R. Haul u. G. Dümbgen, Z. Elektrochem. 66, 636 [1962].

19 E. I. Williams, J. Am. Ceram. Soc. 48, 190 [1965]. 
Dieser Diffusionvorgang kann im Moment noch nicht näher beschrieben werden. Ein Zusammenhang zwischen der Beweglichkeit des Tritiums mit dem Ausheilen bestrahlungsinduzierter Gitterdefekte kann nicht ausgeschlossen werden, erscheint jedoch unwahrscheinlich, da die leichten $\mathrm{T}_{2^{-}}$(und $\mathrm{Li}$-) Ionen eine nur geringe Strahlenschädigung verursachen. Es ist vielmehr wahrscheinlicher, daß die Tritiumatome nach Ionenbeschuß auch im ungeschädigten Material über gewisse Gitterdefekte, statt, wie in Permeationsmessungen, durch die Kanäle der Gitterstruktur wandern. Eine weitere Möglichkeit ist die einer chemischen Wechselwirkung von Tritiumionen mit Sauerstoffionen. Die gemessenen Aktivierungsenergien würden dann neben dem zur Bewegung der Tritiumatome benötigten Energiebetrag noch den Betrag zur

20 C. Mantescu u. T. Costea, Phys. Status Solidi 3, K 290 [1963].

21 H. Cohen u. W. S. Diethorn, Phys. Status Solidi 9, 251 [1965].
Schaffung der Defekte oder den Betrag zur Dissoziation der chemischen Bindung enthalten.

In bisher veröffentlichten Messungen der Diffusion von Tritium in Nicht-Metallen wurde Tritium ebenfalls gewaltsam durch Kernreaktion oder Ionenbeschuß in die Proben eingeführt. Die untersuchten Materialien waren $\mathrm{LiF}^{\mathbf{9}, 20,21}, \mathrm{BeO}^{22,23}$ und Steinmeteoriten ${ }^{24}$. In diesen Fällen wurden ähnlich langsame Diffusionsvorgänge gefunden wie in der vorliegenden Arbeit.

Die vorliegende Arbeit wurde in der Chemistry and Metallurgy Division der Chalk River Nuclear Laboratories ausgeführt. Der Autor dankt besonders dem Personal am Massenseparator für experimentelle Hilfe und Herrn Dr. R. Kelly (CCR Euratom, Ispra) für Diskussionen.

22 A. R. Palmer, D. Roman u. H. J. Whitfield, J. Nucl. Mater. 14, 141 [1964].

23 E. Rothwell, L. L. Wassell u. K. T. Scott, Harwell Report AERE-R-4691 [1964]

24 H. Fechtig u. W. Gentner, Z. Naturforschg. 20 a, 1686[1965].

\title{
Messung der relativen Häufigkeiten der Xenon-Isotope mit dem elektrischen Massenfilter
}

\author{
K. Bruchhausen, S. Gebauer und U. von Zahn \\ Physikalisches Institut der Universität Bonn
}

(Z. Naturforschg. 22 a, $969-972$ [1967] ; eingegangen am 7. März 1967)

\begin{abstract}
The relative abundances of the isotopes of terrestrial xenon were re-determined using a large quadrupole spectrometer. All 11 measured isotopic ratios are in good agreement with the 1950 results of NiER. However, within the error limits a small, but systematic enrichment of the lighter isotopes is noticed in our data compared to NIER's.
\end{abstract}

Es ist seit langem bekannt, daß die aus Meteoriten extrahierten Edelgase eine wesentlich andere Isotopenverteilung haben können als die entsprechenden Edelgase der Erdatmosphäre. Seit einigen Jahren ist die Meßtechnik dieser Untersuchungen so verfeinert, $\mathrm{da} ß$ auch Xenon in diese Messungen einbezogen wird (siehe z. B. Reynolds ${ }^{1}$ oder Marti, Eberhardt und Geiss ${ }^{2}$ ). Als Vergleichswerte für die Zusammensetzung terrestrischen Xenons werden die von $\mathrm{N}_{\text {IER }}{ }^{3}$ benutzt, der für seine Häufigkeitsmessungen ein magnetisches $60^{\circ}$-Spektrometer benutzte. NIER bestimmte die massendiskriminierenden Eigenschaften seiner Apparatur durch Eichmessungen eines Ge-

1 J. H. Reynolds, J. Geophys. Res. 68, 2939 [1963].

2 K. Marti, P. Eberhardt u. J. Geiss, Z. Naturforschg. 21 a, 398 [1966]. misches reiner Argonisotope ${ }^{36} \mathrm{Ar}$ und ${ }^{40} \mathrm{Ar}$, deren Mischungsverhältnis genau bekannt war. Da bisher reine Xenon-Isotope für solche Eichmessungen nicht verfügbar sind, ermittelte NIER seine Korrekturfaktoren für die Xenonmessungen, indem er als sekundären Standard zunächst das Verhältnis ${ }^{128} \mathrm{Xe} /{ }^{136} \mathrm{Xe}$ mit dreifach ionisierten Ionen etwa bei gleichem $e / m$-Verhältnis wie sein primäres Argon-Gemisch bestimmte. Mit den Annahmen, daß die Massendiskriminierung nicht vom Ionisierungsgrad der Atome abhängt und im Bereich der untersuchten Isotope linear von deren Massenzahl abhängt, wurden sodann die gemessenen Xenonhäufigkeiten mit dem am

${ }^{3}$ A. O. Nier, Phys. Rev. 79, 450 [1950]. 